See discussions, stats, and author profiles for this publication at: https://www.researchgate.net/publication/274402864

\title{
Protecting the SWAP operation from general and residual errors by continuous dynamical decoupling
}

Article in Physical Review A · April 2015

DOI: 10.1103/PhysRevA.91.042325 · Source: arXiv

CITATIONS

3

4 authors:

1

F. F. Fanchini

São Paulo State University

45 PUBLICATIONS 2,090 CITATIONS

SEE PROFILE

Baris Cakmak

University of Campinas

17 PUblications 301 Citations

SEE PROFILE

Some of the authors of this publication are also working on these related projects:

Project included by accident View project

Project Ultracold colisions \& trap loss View project
READS

33

Reginaldo de Jesus Napolitano

University of São Paulo

51 PUBLICATIONS 589 CITATIONS

SEE PROFILE

A.O. Caldeira

University of Campinas

85 PUBLICATIONS 8,571 CITATIONS

SEE PROFILE 


\title{
Protecting the $\sqrt{\text { SWAP }}$ operation from general and residual errors by continuous dynamical decoupling
}

\author{
F. F. Fanchini, ${ }^{1, \text { f R R. d. J. Napolitano, }}{ }^{2}$ B. Çakmak,${ }^{3}$ and A. O. Caldeira ${ }^{3}$ \\ ${ }^{1}$ Faculdade de Ciências, UNESP - Universidade Estadual Paulista, Bauru, SP, 17033-360, Brazil \\ ${ }^{2}$ Instituto de Fúsica de São Carlos, Universidade de São Paulo, \\ P.O. Box 369, São Carlos, SP, 13560-970, Brazil \\ ${ }^{3}$ Instituto de Física Gleb Wataghin, Universidade Estadual de Campinas, \\ P.O. Box 6165, Campinas, SP, 13083-970, Brazil
}

(Dated: April 3, 2015)

\begin{abstract}
We study the occurrence of errors in a continuously decoupled two-qubit state during a $\sqrt{\text { SWAP }}$ quantum operation under decoherence. We consider a realization of this quantum gate based on the Heisenberg exchange interaction, which alone suffices for achieving universal quantum computation. Furthermore, we introduce a continuous-dynamical-decoupling scheme that commutes with the Heisenberg Hamiltonian to protect it from the amplitude damping and dephasing errors caused by the system-environment interaction. We consider two error-protection settings. One protects the qubits from both amplitude damping and dephasing errors. The other features the amplitude damping as a residual error and protects the qubits from dephasing errors only. In both settings, we investigate the interaction of qubits with common and independent environments separately. We study how errors affect the entanglement and fidelity for different environmental spectral densities.
\end{abstract}

PACS numbers: 03.67.Pp, 03.67.Lx, 03.67.-a, 03.65.Yz

\section{INTRODUCTION}

Quantum computers use superposition and entanglement of qubits to outperform digital computers [1, 2]. The advent of these machines will unquestionably encompass a radical transformation in the way we simulate quantum-mechanical processes [3], imparting a plethora of new achievements in science and technology. However, to take advantage of the benefits of reliable quantum information processing, we depend on the development of efficient ways to avoid or recover from the errors that, induced by environmental interaction [4], occur in the state of our quantum system.

Accordingly, several strategies to protect quantum information, particularly during the operation of a quantum gate, have been designed, including quantum error correcting codes [5], fault-tolerant quantum computing [6], decoherence-free subspaces [7, 8], etc. One of the most effective methods to protect the state of a quantum system from decoherence is called dynamical decoupling (DD), which has been extensively studied in the literature, both theoretically [9 18] and experimentally [19 25], and is the main subject we focus in this paper. The DD approach is based on applying a sequence of external control pulses to the quantum system to be protected, in order to suppress the errors arising from its coupling with the environment. In other words, we introduce an additional Hamiltonian, called the control Hamiltonian, that acts on the Hilbert space of the system, averaging out the effects of the environmental perturbations.

Alternatively, instead of control pulses, it is also

*Electronic address: fanchini@fc.unesp.br possible to apply continuous external fields to decouple the system from the environmental interactions. This scheme, known as continuous dynamical decoupling (CDD), has attracted a lot of attention in recent years 26 35]. The CDD procedure is more experimentally friendly than pulsed procedures and it also sets a natural stage for the implementation of two-qubit quantum gates [36 38]. For instance, nitrogen vacancy (NV) centers in diamonds have recently been shown to be strong candidates for use in the field of quantum technologies, with possible applications ranging from biological systems to quantum computing protocols [39]. Furthermore, for reducing the damage of environmental perturbations in NV centers, the CDD procedure has again proven to be very important [40].

In this work, we consider CDD of a two-qubit system going through a $\sqrt{\mathrm{SWAP}}$ operation while interacting with a bosonic environment. While Refs. [36 38] treat the case of magnetic noise, where the couplings between the qubits and their environment are orders of magnitude stronger than the interqubit interaction, here we consider the case in which the two-qubit gate interaction is stronger than the perturbations by the environment. We obtain a simple control prescription which allows us to prove the effectiveness of our method in a realistic decoherence model. The effect of the environment is simulated by two different quantum channels amplitude damping and dephasing - simultaneously and independently coupled to the qubits with different coupling strengths. We also study the effects of residual errors when the CDD protection is supplied just against the predominant error source, i.e., the one with the strongest coupling. In the present context, we consider the residual error as arising from the amplitude damping channel, while the qubits are dynamically decoupled from 
dephasing. We adopt the concurrence [41] and the fidelity as the figures of merit of the CDD procedure. We show that the adopted CDD scheme provides nearly full protection against environmental effects when both error mechanisms are present and, for a residual amplitudedamping environment, a super-ohmic spectral density of states is more destructive than an ohmic one. Furthermore, in the absence of the CDD, we see that, in the case of a common environment for the qubits, both entanglement and fidelity decay more slowly than in the case of independent environments.

The present paper is organized as follows. Section II reviews the CDD procedure for the case of a $\sqrt{\text { SWAP }}$ quantum gate. Section III describes our model to simulate the error sources. Section IV shows the results for the CDD protection of the $\sqrt{\mathrm{SWAP}}$ from both amplitude damping and dephasing, and from dephasing only, when amplitude damping is treated as a residual error channel. Finally, a conclusion is presented in Sec. V. The detailed solution of the master equation, that gives the dynamics of the reduced density matrix of the system, is presented in the Appendix.

\section{THE MODEL AND CONTINOUS DYNAMICAL DECOUPLING}

To illustrate our protective scheme, we begin by assuming that the interaction Hamiltonian between the qubit system and the rest of the universe is of the form

$$
H_{\text {int }}=\mathbf{B}^{(1)} \cdot \boldsymbol{\sigma}^{(1)}+\mathbf{B}^{(2)} \cdot \boldsymbol{\sigma}^{(2)},
$$

where $\mathbf{B}^{(s)}=\sum_{m=1}^{3} B_{m}^{(s)} \hat{\mathbf{x}}_{m}$, for $s=1,2$, with $\hat{\mathbf{x}}_{1} \equiv \hat{\mathbf{x}}$, $\hat{\mathbf{x}}_{2} \equiv \hat{\mathbf{y}}, \hat{\mathbf{x}}_{3} \equiv \hat{\mathbf{z}}$, and $B_{m}^{(s)}$, for $s=1,2$ and $m=1,2,3$, are Hermitian operators that act on the environmental Hilbert space. The main approach of the dynamical decoupling method [9 18] in order to reduce errors on the system, is to eliminate the effect of the interaction Hamiltonian by an external control Hamiltonian. Mathematically, such a condition can be written as

$$
\int_{0}^{t_{c}} U_{c}^{\dagger}(t) H_{\mathrm{int}} U_{c}(t) d t=0
$$

where $U_{c}(t)$ is the time evolution operator associated with the control Hamiltonian $H_{c}$ and $t_{c}=2 \pi / \omega$. Equation (2) results from a Magnus expansion [42] used to describe the total interaction-picture evolution operator, in the limit in which $t_{c} \rightarrow 0$. In this limit, only the first term of the expansion, given by the integral in Eq. (2), is, in general, non-zero. Thus, in this ideal circumstance, by imposing Eq. (2) we are ensuring the complete elimination of the perturbing interactions. Here, however, we use Eq. (2) as a mere guide to develop the present approach, for our focus is on the realistic situation of a finite $t_{c}$. In the present paper, we will gauge numerically the efficacy of the resulting approximate method.
In order to control the intensity of the exchange interaction, possible candidates for the physical qubits should be, for example, properly built tunable charge qubits [43]. Although, in this particular case, physical reasoning leads us to assume that each qubit is coupled to its own environment, we shall, for the sake of completeness, also study the case of a common environment. For our present purposes, we assume that the particular form of Eq. (11) is, in the case of a common environment, given by

$$
H_{\text {int }}=\left(\boldsymbol{\sigma}^{(1)}+\boldsymbol{\sigma}^{(2)}\right) \cdot\left(\boldsymbol{\lambda} B+\boldsymbol{\lambda}^{*} B^{\dagger}\right),
$$

where $\boldsymbol{B}^{(s)}=\boldsymbol{\lambda} B+\boldsymbol{\lambda}^{*} B^{\dagger}$, for $s=1,2, \boldsymbol{\lambda}$ is an arbitrary complex three-dimensional vector, and $B$ is a scalar operator that acts on the environmental Hilbert space. However, when the qubits are physically located sufficiently far apart, as for tunable charge qubits [43], it is reasonable to suppose that their individual surroundings act as uncorrelated, independent environments. In that case, the particular form we assume for Eq. (11) is written as

$$
\begin{aligned}
H_{i n t} & =\boldsymbol{\sigma}^{(1)} \cdot\left(\boldsymbol{\lambda}^{(1)} B^{(1)}+\boldsymbol{\lambda}^{(1)^{*}} B^{(1)^{\dagger}}\right) \\
& +\boldsymbol{\sigma}^{(2)} \cdot\left(\boldsymbol{\lambda}^{(2)} B^{(2)}+\boldsymbol{\lambda}^{(2)^{*}} B^{(2)^{\dagger}}\right)
\end{aligned}
$$

where $B^{(s)}$, for $s=1,2$, acts on the environmental Hilbert space of the $s$-th qubit, and $\boldsymbol{\lambda}^{(s)}$ is an arbitrary complex three-dimensional vector for $s=1,2$.

In the interaction picture associated with $H_{c}(t)$, the total Hamiltonian can be written as

$$
H(t)=H_{0}+H_{E}+U_{c}^{\dagger}(t) H_{\mathrm{int}} U_{c}(t),
$$

where $H_{0}$ is the Hamiltonian that performs the desired gate operation we want to protect and $H_{E}$ is the environmental Hamiltonian satisfying $U_{c}^{\dagger}(t) H_{E} U_{c}(t)=$ $H_{E}$. We represent the environment of each qubit as a thermal bath of harmonic oscillators. In the case of a common environment for both qubits, we consider $H_{E}=\sum_{k} \omega_{k} a_{k}^{\dagger} a_{k}$, where $\omega_{k}$ is the frequency of the $k$-th normal mode of the common environment, and $a_{k}$ and $a_{k}^{\dagger}$ are the annihilation and creation operators, respectively. In the case of two independent and identical environments, instead of the above we take $H_{E}=\sum_{s=1}^{2} \sum_{k} \omega_{k} a_{k}^{(s)^{\dagger}} a_{k}^{(s)}$, where $\omega_{k}$ is the frequency of the $k$-th normal mode of the $s$-th qubit environment, and $a_{k}^{(s)}$ and $a_{k}^{(s)^{\dagger}}$ are, respectively, the corresponding annihilation and creation operators. The frequency $\omega_{k}$ is the same for both independent and identical environments. Accordingly, we take $B_{m}^{(s)}=$ $\sum_{k}\left(\lambda_{m} g_{k}^{*} a_{k}^{(s)}+\lambda_{m}^{*} g_{k} a_{k}^{(s) \dagger}\right)$, where $g_{k}$ are coupling constants.

In a previous work, we have shown that it is possible to use a continuously-applied external field to protect entangled states from errors caused by the unavoidable interactions between the qubit system and its environment 31]. The question naturally arises as to whether it is also 
possible to protect an entangling operation. In the following, we show that, using the very same external-field configuration of Ref. 31], we can prevent errors from occurring during the application of a $\sqrt{\text { SWAP }}$ quantum gate to a two-qubit state under decoherence.

An ideal $\sqrt{\text { SWAP }}$ gate is obtained by the Heisenberg coupling between two qubits, whose dynamics is governed by the Hamiltonian

$$
H_{0}=J \boldsymbol{\sigma}^{(1)} \cdot \boldsymbol{\sigma}^{(2)},
$$

where we use $\hbar=1$ throughout, $J$ is the exchange constant, and, for $s=1,2, \sigma^{(s)}=\hat{\mathbf{x}} \sigma_{x}^{(s)}+\hat{\mathbf{y}} \sigma_{y}^{(s)}+\hat{\mathbf{z}} \sigma_{z}^{(s)}$, where $\sigma_{x}^{(s)}, \sigma_{y}^{(s)}$, and $\sigma_{z}^{(s)}$ are the Pauli matrices acting on qubit s. Remarkably, it has been shown that the Heisenberg interaction alone is sufficient for universal quantum computation, without the need of supplementary single-qubit operations 44 46]. Thus, a protective scheme for quantum gates based on this interaction, such as the $\sqrt{\text { SWAP }}$ operation, is of fundamental importance. Our results may find important applications in various experimental settings, such as double quantum dots 47] and neutral atoms in optical lattices [48], where the exchange interaction between two-qubits can be realized.

It is possible to protect the considered quantum gate actualized by the Hamiltonian in Eq. (6) by the control Hamiltonian of the form

$$
H_{c}(t)=\boldsymbol{\Omega}(t) \cdot\left(\boldsymbol{\sigma}^{(1)}+\boldsymbol{\sigma}^{(2)}\right) .
$$

In order for the evolution operator associated with the control Hamiltonian to satisfy Eq. (2) we must have [31]:

$$
U_{c}(t)=U^{(2)}(t) U^{(1)}(t)=U^{(1)}(t) U^{(2)}(t),
$$

since $\boldsymbol{\sigma}^{(1)}$ and $\boldsymbol{\sigma}^{(2)}$ commute, where

$$
U^{(s)}(t)=\exp \left(-i \omega t n_{x} \sigma_{x}^{(s)}\right) \exp \left(-i \omega t n_{z} \sigma_{z}^{(s)}\right),
$$

for $s=1,2$.

Equations (8) and (9) imply the following external field configuration:

$$
\boldsymbol{\Omega}(t)=\hat{\mathbf{x}} n_{x} \omega+n_{z} \omega\left[\hat{\mathbf{z}} \cos \left(n_{x} \omega t\right)-\hat{\mathbf{y}} \sin \left(n_{x} \omega t\right)\right] .
$$

Here $\omega=2 \pi / t_{c}, n_{x}$ and $n_{z} \neq n_{x}$ are non-zero integers, and $t_{c}$ is a constant. Such a field configuration is a combination of a static field along the $x$ axis and a rotating field in the $y z$ plane, and it is able to protect the evolution described by the Hamiltonian of Eq. (6) from the effects of a general class of errors. We can modify this field arrangement to be protective solely against a dephasing channel by setting $n_{z}=0$ in Eq. (10). In this case we only have a static field along the $x$ axis, given by $\boldsymbol{\Omega}(t)=\hat{\mathbf{x}} n_{x} \omega$, which is simpler than the field arrangement given in Eq. (10). Moreover, the field is supposed to be spatially uniform in the neighborhood surrounding both qubits, since it is not necessary to address each qubit independently.
Because Eq. (6) is a scalar product, it is invariant under rotations and

$$
U_{c}^{\dagger}(t) H_{0} U_{c}(t)=H_{0} .
$$

This property of the Heisenberg interaction tremendously simplifies the quantum operations executed under the protection by the CDD, due to the fact that the intended gate operation remains intact under the action of the control fields. Without this rotational invariance, we would have to proceed as in Ref. [30] and introduce an auxiliary rotating reference frame which complicates the procedure. Furthermore, this invariance has another important property: with the exact same field arrangement that preserves a quantum memory, we can also protect the quantum-gate operation. Being able to protect the gate operation without the necessity of field reconfiguration is a tremendous simplification and certainly improves the prospects for experimental realization.

\section{PROTECTING THE $\sqrt{\text { SWAP }}$ GATE}
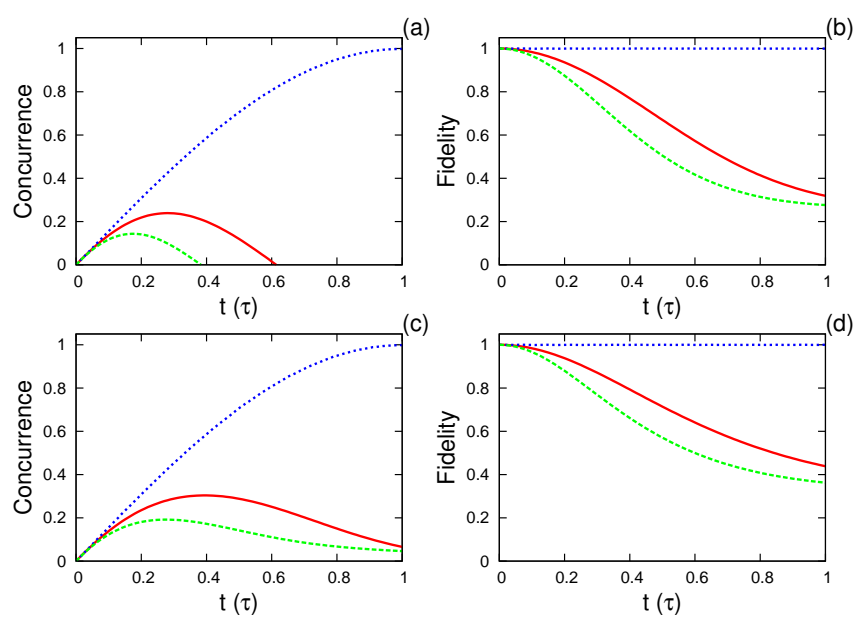

FIG. 1: (Color Online) Amplitude Damping plus Dephasing: In figures (a) and (b) we show, respectively, the concurrence and fidelity for independent environments and, in figures (c) and (d), for the case of a common environment. The dotted (blue) line represents the dynamics of a $\sqrt{\text { SWAP }}$ quantum gate with protection. The solid (red) and dashed (green) lines represent the dynamics without protection for ohmic and super-ohmic spectral densities, respectively.

In order to illustrate our protective scheme, we take a Heisenberg interaction strength $J=\pi / 8$ (c.f. Eq. (6) ) and a cut-off frequency for the spectral density, $J(\omega)=\eta \frac{\omega^{s}}{\omega_{c}^{s-1}} \exp \left(-\omega / \omega_{c}\right)$, given by $\omega_{c} \tau=2 \pi$, as explained in the Appendix, where $\tau=10^{-9} \mathrm{~s}$. We consider that the qubits interact with independent and common environments which are, in both cases, assumed to be at $T=0.2 \mathrm{~K}$, with a coupling constant $\eta=1 / 20$, as specified in the Appendix. We investigate each environment model with ohmic $(\mathrm{s}=1)$ and super-ohmic $(\mathrm{s}=3)$ spectral 
densities. For the protected cases, we set the external field parameters as $n_{x}=28 \pi / \tau$ and $n_{z}=14 \pi / \tau$ (c.f. Eq. (10)). We choose the initial state of the system to be $\rho(0)=|\uparrow \downarrow\rangle\langle\uparrow \downarrow|$. Note that the initial state is a product state and, in the absence of any decoherence, application of the $\sqrt{\text { SWAP }}$ gate to this initial state will create a maximally entangled state.

\section{A. Amplitude damping and dephasing errors}

In Fig. (11) we show the fidelity and concurrence of our two-qubit system, for the protected and unprotected cases, during the application of the $\sqrt{\text { SWAP }}$ operation, when both amplitude damping and dephasing errors are present. We observe that, in the protected cases, the fidelity remains near unity during the whole time evolution, as opposed to the unprotected cases, where the fidelity decays for both ohmic and super-ohmic spectra, with a higher decay rate in the super-ohmic case. However, since the value of the concurrence is near unity at the end of the time evolution of the protected cases, we conclude that the entangling operation is successfully carried out. In fact, in the protected cases shown, higher values of fidelity and concurrence can be obtained for higher values of $n_{x}$ and $n_{z}$. However, the same is not true for the unprotected gate operation. For independent environments, the concurrence presents a peak, but then decays to zero with a lower maximum value and faster decay rate for the super-ohmic than for the ohmic spectrum. Finally, we see that the concurrence again presents a peak, followed by decay to a low but finite value, in the case of a common environment.

\section{B. Amplitude damping as a residual error}

In Fig. (2) we present the fidelity and concurrence of our two-qubit system, during the application of a $\sqrt{\text { SWAP }}$ gate, protected only from dephasing, while the amplitude damping channel is left open as a residual error channel. We maintain the same external field configuration introduced in the beginning of this section, but set $n_{z}=0$, which makes our system vulnerable to the residual errors. Interestingly, we see that whether our two qubits interact independent or collectively with the environment has very little effect on the fidelity and concurrence. In both cases of interaction with the environment, the residual errors cause less damage to the system for the ohmic than for the super-ohmic spectral density.

In Fig. (3) we show the concurrence and fidelity at the end of the quantum gate operation, i.e., at $t=\tau$, as functions of the amplitude-damping coupling constant $\lambda$ (see Eqs. (3) and (4)). For all possible cases of environmental spectral density and interaction, the concurrence and fidelity decrease linearly as functions of the coupling constant. While the case of a common environment with an ohmic spectral density presents the highest decay rate, the case of independent environments with ohmic spectra shows the lowest decay rates, as functions of $\lambda$. However, it is important to note that, regarding these functions, there is little difference between the case of a common environment and that of two independent environments. A plausible argument to explain this behaviour would be to recall the fact that in our case qubit-qubit coupling is stronger than qubits' coupling to the environment. Therefore, any environmental effect on one of the qubits is quickly felt by the other qubit for both common and independent environments.
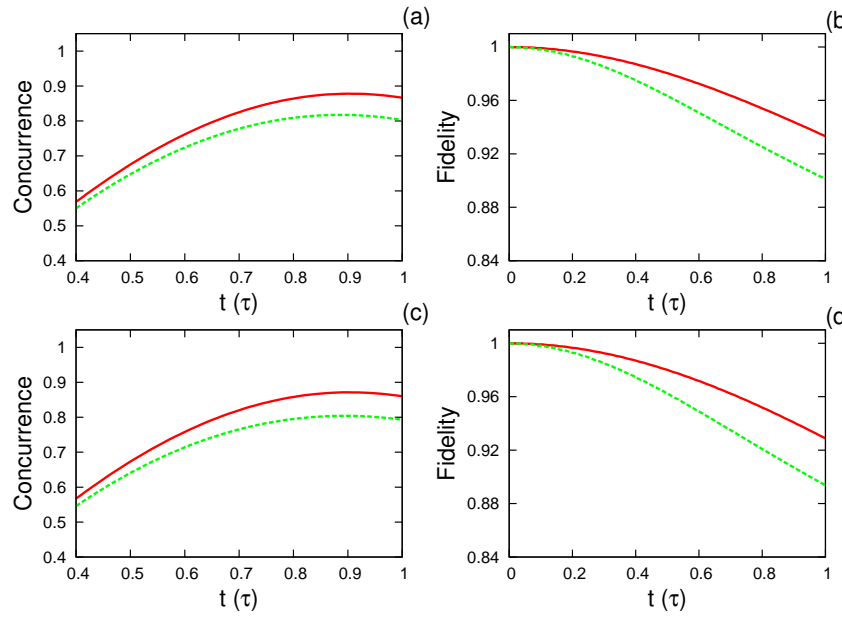
(d)

FIG. 2: (Color Online) Amplitude damping as a residual error channel: In figures (a) and (b) we show, respectively, the concurrence and fidelity for independent environments and, in figures (c) and (d), for the case of a common environment. The solid (red) and dashed (green) lines represent the dynamics of a $\sqrt{\text { SWAP }}$ quantum gate for ohmic and super-ohmic environments, respectively.

\section{CONCLUSION}

In summary, we have presented a CDD strategy to protect, from general and residual errors, a $\sqrt{\text { SWAP }}$ quantum gate, which is an entangling operation realizable using the Heisenberg exchange interation. The gate operation is applied to a two-qubit system and the errors are introduced by amplitude-damping and dephasing channels, resulting from interactions with bosonic environments. We consider both common and independent environments, together with ohmic and super-ohmic environmental spectral densities. We quantify the success of protection by looking at the fidelity and concurrence of the system during the time of gate operation. When both error mechanisms are present and no protection is supplied, we observe that the case of two independent environments with super-ohmic spectral densities is the most harmful. However, we have shown that our protection scheme works very well against these errors, keeping the system at high fidelity and letting the entangling gate operation perform successfully. We have also considered 
(a)
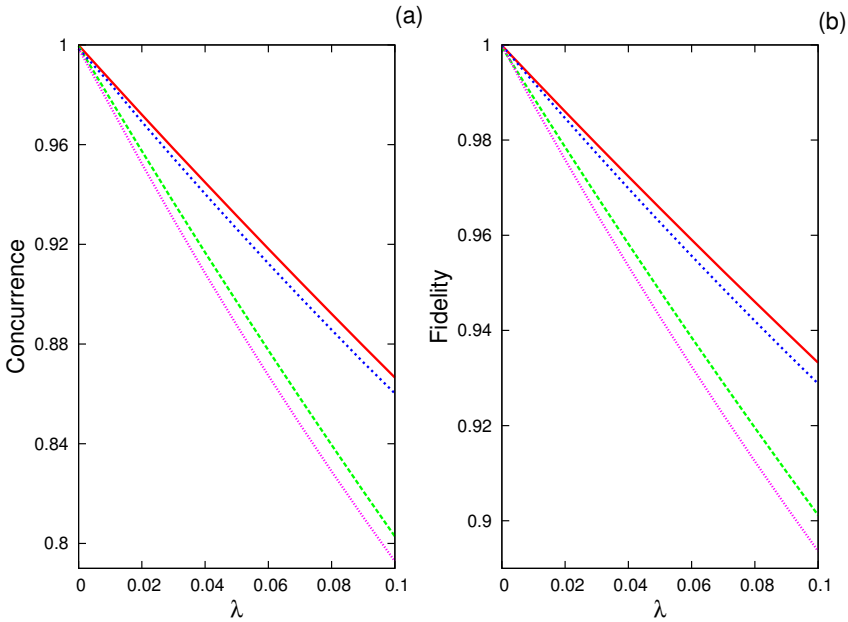

FIG. 3: (Color Online) The concurrence (a) and fidelity (b) at the end of the quantum gate operation, i.e., at $t=\tau$, as functions of the coupling constant, $\lambda$, of the residual error channel. The solid (red) line represents the case of independent environments with ohmic spectra, the dotted (blue) line represents the case of a common environment with an ohmic spectrum, the dashed (green) line represents the case of independent environments with super-ohmic spectra, and the purple (closely-dotted) line represents the case of a common environment with a super-ohmic spectrum.

a residual error setting, where only dephasing errors are protected, while amplitude-damping errors are allowed to affect the system. In this case, we have seen that independent or common environmental interactions show little difference and the case of a super-ohmic spectral density continues to be more harmful to the gate operation as in the previous case. Furthermore, our CDD scheme uses the same external field configuration in the dynamic as in the static case, which is an important property for experimental applications.

Finally, we would like to say a few words about the dependence of the concurrence and fidelity on the spectral function of separable and common environments. To start with, let us address the issue of separability.

As a matter of fact, this dependence only shows up in the rate of change of those quantities. Separable environments seem to destroy them faster than the common ones. Although we are not going to provide any detailed explanation for this fact, we can argue that quantum coherence properties between two qubits are more likely to be preserved by the presence of a common environment since it could mediate, at least in some cases, an indirect effective coupling between them. In other words, in common environments there might be the possibility of existence of some cooperative effect on top of the deleterious effects present in any coupling to general environments. On the other hand, separable environments would always act independently on each qubit giving them very little chance to preserve or develop any quantum mechanical coherence.
As for the spectral function dependence, the stronger effect of superohmic environments as compared to that of ohmic environments can be simply understood in terms of time scales. It is a very simple matter for the reader to convince him or herself that the general spectral function $J(\omega)$ defined in the beginning of section III has a maximum at $\omega_{m}^{(s)}=s \omega_{c}$ whose value is $J_{\max }^{(s)} \equiv J\left(\omega_{m}^{(s)}\right)=$ $\eta \omega_{c}(s / e)^{s}$. Therefore, we see that for superohmic environments (with $s=3) J(\omega)$ is peaked at $3 \omega_{c}$ with its maximum value given by $J_{m}^{(3)} \approx 1.1 \eta \omega_{c}$ whereas these values are, respectively, $\omega_{c}$ and $J_{m}^{(1)} \approx 0.4 \eta \omega_{c}$ for ohmic environments. Another important point to be observed is that since we are interested in protection schemes, and consequently testing our system for times in the interval $0<t<\tau=2 \pi / \omega_{c}$, the relevant frequency range for our analysis is $\omega>\omega_{c} / 2 \pi$.

Therefore we see that as time evolves from $t=0$, the deleterious effects of the superohmic environment start to take place earlier than those of the ohmic environment because the spectral weight of the former is more pronounced at a higher frequency than that of the latter. In other words, it is the high frequency behavior of the spectral function which dominates any phenomena at this time scale.

At this point one should naturally argue that our reasoning does not make much sense for the present problem because the form established for the spectral function $J(\omega)$ is actually appropriate for dealing with phenomena at very long time scales $\left(t \gg 1 / \omega_{c}\right)$ which are dominated by the low frequency behavior of $J(\omega)$. The cutoff frequency $\omega_{c}$ is a characteristic frequency of the environment which fixes the time scale of the problem. Nevertheless, we can still sustain our results if we take them as an indication that no matter what bath we have, it is its high frequency behavior that matters in this case. This means that if one is really interested in a more quantitative analysis of the problem, a more detailed account of the environment's high frequency behavior is in order.

\section{ACKNOWLEDGEMENTS}

This work has been partly supported by "Fundação de Amparo à Pesquisa do Estado de São Paulo (FAPESP)", Brazil, project numbers 12/50464-0 and 2014/217925 , and by "Conselho Nacional de Desenvolvimento Científico e Tecnológico (CNPq)", Brazil, through the "Instituto Nacional de Ciência e Tecnologia em Informação Quântica (INCT-IQ)". 


\section{Appendix: Solution of the master equation}

Starting from $H(t)$, the Hamiltonian in the interaction picture is written as

$$
H_{I}(t)=\sum_{s=1}^{2} \sum_{m=1}^{3} \sum_{n=1}^{3} R_{m, n}(t) E_{m}^{(s)}(t) \widetilde{\sigma}_{n}^{(s)}(t)
$$

where $\sigma_{1}^{(s)} \equiv \sigma_{x}^{(s)}, \sigma_{2}^{(s)} \equiv \sigma_{y}^{(s)}, \sigma_{3}^{(s)} \equiv \sigma_{z}^{(s)}, \widetilde{\sigma}_{n}^{(s)}(t)=$ $U_{0}^{\dagger}(t) \sigma_{n}^{(s)} U_{0}(t)$, for $s=1,2$ and $n=1,2,3$, with $U_{0}(t)=$ $\exp \left(-i H_{0} t\right)$. We have used Eq. (10) and defined the operators $E_{m}^{(s)}(t)=U_{E}^{\dagger}(t) B_{m}^{(s)} U_{E}(t)$, for $s=1,2$ and $m=1,2,3$, with $U_{E}(t)=\exp \left(-i H_{E} t\right)$. The quantities $U_{c}^{\dagger}(t) \sigma_{m}^{(s)} U_{c}(t)=\sum_{n=1}^{3} R_{m, n}(t) \sigma_{n}^{(s)}$, for $s=1,2$ and $m=1,2,3$, are rotations of $\sigma_{m}^{(s)}$, whose matrix elements, $R_{m, n}(t)$, are real functions of time. We proceed as in Ref. [31] and assume that the absolute temperature is the same in the surroundings of both qubits and these qubits, as well as their respective environments, are identical. We then write down the master equation for the two-qubit reduced density matrix, $\rho_{I}(t)$, in the Born approximation:

$$
\frac{d \rho_{I}(t)}{d t}=\sum_{s, s^{\prime}=1}^{2} \sum_{n, n^{\prime}=1}^{3} \int_{0}^{t} d t^{\prime}\left\{\mathcal{D}_{n, n^{\prime}}^{\left(s, s^{\prime}\right)}\left(t, t^{\prime}\right)\left[\widetilde{\sigma}_{n}^{(s)}(t), \rho_{I}(t) \widetilde{\sigma}_{n^{\prime}}^{\left(s^{\prime}\right)}\left(t^{\prime}\right)\right]+\left[\mathcal{D}_{n, n^{\prime}}^{\left(s, s^{\prime}\right)}\left(t, t^{\prime}\right)\right]^{*}\left[\widetilde{\sigma}_{n^{\prime}}^{\left(s^{\prime}\right)}\left(t^{\prime}\right) \rho_{I}(t), \widetilde{\sigma}_{n}^{(s)}(t)\right]\right\}
$$

where we have defined the coefficients

$$
\mathcal{D}_{n, n^{\prime}}^{\left(s, s^{\prime}\right)}\left(t, t^{\prime}\right)=\sum_{m=1}^{3} \sum_{m^{\prime}=1}^{3} R_{m, n}(t) R_{m^{\prime}, n^{\prime}}\left(t^{\prime}\right) C_{m, m^{\prime}}^{\left(s, s^{\prime}\right)}\left(t, t^{\prime}\right)
$$

for $n, n^{\prime}=1,2,3$ and $s, s^{\prime}=1,2$, and

$$
C_{m, m^{\prime}}^{\left(s, s^{\prime}\right)}\left(t, t^{\prime}\right)=\operatorname{Tr}_{E}\left\{E_{m}^{(s)}(t) \rho_{E} E_{m^{\prime}}^{\left(s^{\prime}\right)}\left(t^{\prime}\right)\right\}
$$

for $m, m^{\prime}=1,2,3$ and $s, s^{\prime}=1,2 . \quad C_{m, m^{\prime}}^{\left(s, s^{\prime}\right)}\left(t, t^{\prime}\right)$ is the correlation function between components $m$ and $m^{\prime}$ of environmental operators calculated at the same qubit position, as explained in Ref. 31. Here, $\operatorname{Tr}_{E}$ denotes the trace over the environmental degrees of freedom. The operators $\widetilde{\sigma}_{n}^{(s)}(t)$, for $s=1,2$ and $n=1,2,3$, can be explicitly obtained as the components of the following vector relations:

$$
\begin{array}{r}
U_{0}^{\dagger}(t) \boldsymbol{\sigma}^{(1)} U_{0}(t)=a(t) \boldsymbol{\sigma}^{(1)}+b(t) \boldsymbol{\sigma}^{(2)} \\
-c(t)\left(\boldsymbol{\sigma}^{(1)} \times \boldsymbol{\sigma}^{(2)}\right),
\end{array}
$$

and

$$
\begin{aligned}
U_{0}^{\dagger}(t) \boldsymbol{\sigma}^{(2)} U_{0}(t)= & a(t) \boldsymbol{\sigma}^{(2)}+b(t) \boldsymbol{\sigma}^{(1)} \\
& -c(t)\left(\boldsymbol{\sigma}^{(2)} \times \boldsymbol{\sigma}^{(1)}\right),
\end{aligned}
$$

where $a(t)=[1+\cos (4 J t)] / 2, b(t)=[1-\cos (4 J t)] / 2$, and $c(t)=\sin (4 J t) / 2$. The environmental density matrix,
$\rho_{E}$, is taken as the one for a canonical ensemble constituting a thermal bath, that is, $\rho_{E}=\frac{1}{Z} \exp \left(-\beta H_{E}\right)$,where $Z$ is the partition function, $Z=\operatorname{Tr}_{E}\left[\exp \left(-\beta H_{E}\right)\right]$. Here, $\beta=1 / k_{B} T, k_{B}$ is the Boltzmann constant, and $T$ is the absolute temperature of the environment.

We can also write the correlation function as

$$
C_{m, m^{\prime}}^{\left(s, s^{\prime}\right)}\left(t, t^{\prime}\right)=\Gamma^{\left(s, s^{\prime}\right)} \operatorname{Tr}_{E}\left\{E_{m}^{(s)}(t) \rho_{E} E_{m^{\prime}}^{(s)}\left(t^{\prime}\right)\right\},
$$

where $\Gamma^{\left(s, s^{\prime}\right)}=1$ for the case of a single, common environment, in which case the environmental operators $E_{m}^{(s)}(t)$ are independent of $s$, and $\Gamma^{\left(s, s^{\prime}\right)}=\delta_{s, s^{\prime}}$ for the case of two identical, uncorrelated environments. Since we have

$$
E_{m}^{(s)}(t)=\sum_{k}\left[\lambda_{m} g_{k}^{*} a_{k}^{(s)} e^{-i \omega_{k} t}+\lambda_{m}^{*} g_{k} a_{k}^{(s) \dagger} e^{+i \omega_{k} t}\right]
$$

and, therefore,

$$
\begin{array}{r}
\operatorname{Tr}_{E}\left\{E_{m}^{(s)}(t) \rho_{E} E_{m^{\prime}}^{(s)}\left(t^{\prime}\right)\right\}=\lambda_{m} \lambda_{m^{\prime}}^{*} \sum_{k}\left|g_{k}\right|^{2} n_{k} e^{-i \omega_{k}\left(t-t^{\prime}\right)} \\
+\lambda_{m}^{*} \lambda_{m^{\prime}} \sum_{k}\left|g_{k}\right|^{2}\left(1+n_{k}\right) e^{i \omega_{k}\left(t-t^{\prime}\right)},
\end{array}
$$

where $n_{k}=1 /\left[\exp \left(\beta \omega_{k}\right)-1\right]$, we obtain

$$
\frac{d \rho_{I}(t)}{d t}=\sum_{s, s^{\prime}=1}^{2} \int_{0}^{t} d t^{\prime} \mathcal{T}_{1}^{\left(s, s^{\prime}\right)}\left(t-t^{\prime}\right)\left[\mathcal{R}^{(s)}(t), \rho_{I}(t)\left[\mathcal{R}^{\left(s^{\prime}\right)}\left(t^{\prime}\right)\right]^{\dagger}\right]+\sum_{s, s^{\prime}=1}^{2} \int_{0}^{t} d t^{\prime} \mathcal{T}_{2}^{\left(s, s^{\prime}\right)}\left(t-t^{\prime}\right)\left[\left[\mathcal{R}^{(s)}(t)\right]^{\dagger}, \rho_{I}(t) \mathcal{R}^{\left(s^{\prime}\right)}\left(t^{\prime}\right)\right]
$$




$$
+\sum_{s, s^{\prime}=1}^{2} \int_{0}^{t} d t^{\prime}\left[\mathcal{T}_{1}^{\left(s, s^{\prime}\right)}\left(t-t^{\prime}\right)\right]^{*}\left[\mathcal{R}^{\left(s^{\prime}\right)}\left(t^{\prime}\right) \rho_{I}(t),\left[\mathcal{R}^{(s)}(t)\right]^{\dagger}\right]+\sum_{s, s^{\prime}=1}^{2} \int_{0}^{t} d t^{\prime}\left[\mathcal{T}_{2}^{\left(s, s^{\prime}\right)}\left(t-t^{\prime}\right)\right]^{*}\left[\left[\mathcal{R}^{\left(s^{\prime}\right)}\left(t^{\prime}\right)\right]^{\dagger} \rho_{I}(t), \mathcal{R}^{(s)}(t)\right]
$$

where

$$
\begin{gathered}
\mathcal{R}^{(s)}(t)=\sum_{m=1}^{3} \sum_{n=1}^{3} \lambda_{m} R_{m, n}(t) \widetilde{\sigma}_{n}^{(s)}(t), \\
\mathcal{T}_{1}^{\left(s, s^{\prime}\right)}(t)=\Gamma^{\left(s, s^{\prime}\right)} \sum_{k}\left|g_{k}\right|^{2} n_{k} \exp \left(-i \omega_{k} t\right),
\end{gathered}
$$

and

$$
\mathcal{T}_{2}^{\left(s, s^{\prime}\right)}(t)=\Gamma^{\left(s, s^{\prime}\right)} \sum_{k}\left|g_{k}\right|^{2}\left(1+n_{k}\right) \exp \left(i \omega_{k} t\right) .
$$

In the limit the number of environmental normal modes per unit frequency becomes very large, we define a spectral density as $J(\omega)=\sum_{k}\left|g_{k}\right|^{2} \delta\left(\omega-\omega_{k}\right)$, with $\omega \in[0, \infty)$, and interpret the summations in $\mathcal{T}_{1}^{\left(s, s^{\prime}\right)}(t)$ and $\mathcal{T}_{2}^{\left(s, s^{\prime}\right)}(t)$ as integrals over $\omega$ :

$$
\mathcal{T}_{1}^{\left(s, s^{\prime}\right)}(t)=\Gamma^{\left(s, s^{\prime}\right)} \int_{0}^{\infty} d \omega J(\omega) \frac{\exp (-i \omega t)}{\exp (\beta \omega)-1}
$$

and

$$
\mathcal{T}_{2}^{\left(s, s^{\prime}\right)}(t)=\left[\mathcal{T}_{1}^{\left(s, s^{\prime}\right)}(t)\right]^{*}+\Gamma^{\left(s, s^{\prime}\right)} \int_{0}^{\infty} d \omega J(\omega) \exp (i \omega t),
$$

for $s, s^{\prime}=1,2$. Here we assume a cutoff frequency $\omega_{c}$, and write $J(\omega)=\eta\left(\omega^{s} / \omega_{c}^{s-1}\right) \exp \left(-\omega / \omega_{c}\right)$, where $\eta$ is a dimensionless coupling constant, and the values of $s$ specify the two kinds of environmental spectral density we treat: ohmic $(s=1)$ and super-ohmic $(s>1)$.
[1] D. Deutsch and R. Jozsa, Proc. R. Soc. London Ser. A 439, 553 (1992).

[2] P. W. Shor, in Proceedings, 35th Annual Symposium on the Foundations of Computer Science, edited by S. Goldwasser (IEEE Computer Society Press, Los Alamitos, 1994), p. 124.

[3] R. P. Feynman, Int. J. Theor. Phys. 21, 467 (1982).

[4] W. H. Zurek, Phys. Today 44, 36 (1991); arXiv:quant-ph/0306072 1 (2003).

[5] A. M. Steane, Phys. Rev. Lett. 77, 793 (1996).

[6] P. W. Shor, in 37th Symposium on Foundations of Computing, IEEE Computer Society Press, 1996, pp. 56-65; J. Preskill in "Introduction to Quantum Computation edited by H.-K. Lo, S. Popescu, and T. P. Spiller (World Scientific, 1998).

[7] D. A. Lidar, I. L. Chuang, and K. B. Whaley, Phys. Rev. Lett. 81, 2594 (1998).

[8] D. A. Lidar and K. B. Whaley, in Irreversable Quantum Dynamics edited by F. Benatti and R. Floreanini.

[9] L. Viola and S. Lloyd, Phys. Rev. A 58, 2733 (1998).

[10] L. Viola, E. Knill, and S. Lloyd, Phys. Rev. Lett. 82, 2417 (1999).

[11] P. Zanardi, Phys. Lett. A 258, 77 (1999).

[12] M. S. Byrd and D. A. Lidar, Quant. Inf. Proc. 1, 19 (2002).

[13] P. Facchi, S. Tasaki, S. Pascazio, H. Nakazato, A. Tokuse, and D. A. Lidar, Phys. Rev. A 71, 022302 (2005).

[14] K. Khodjasteh and D. A. Lidar, Phys. Rev. A 78, 012355 (2008).

[15] K. Khodjasteh and L. Viola, Phys. Rev. Lett. 102, 080501 (2009).

[16] K. Khodjasteh and L. Viola, Phys. Rev. A 80, 032314 (2009).

[17] K. Khodjasteh, D. A. Lidar, and L. Viola, Phys. Rev. Lett. 104, 090501 (2010).
[18] D. A. Lidar, Adv. Chem. Phys. 154, 295 (2014).

[19] M. J. Biercuk, H. Uys, A. P Vandevender, N. Shiga, W. M. Itano, and J. J. Bollinger, Nature 458, 996 (2009).

[20] J. Du, X. Rong, N. Zhao, Y. Wang, J. Yang, and R. B. Lau, Nature 461, 1265 (2009).

[21] S. Damodarakurup, M. Lucamarini, G. Di Giuseppe, D. Vitali, and P. Tombesi, Phys. Rev. Lett. 103, 040502 (2009).

[22] G. de Lange, Z. H. Wang, D. Ristè, V. V. Dobrovitski, and R. Hanson, Science 330, 60 (2010).

[23] A. M. Souza, G. A. Álvarez, and D. Suter, Phys. Rev. Lett. 106, 240501 (2011).

[24] B. Naydenov, F. Dolde, L. T. Hall, C. Shin, H. Fedder, L. C. L. Hollenberg, F. Jelezko, and J. Wrachtrup, Phys. Rev. B 83, 081201(R).

[25] T. van der Sar, Z. H. Wang, M. S. Blok, H. Bernien, T. H. Taminiau, D. M. Toyli, D. A. Lidar, D. D. Awschalom, R. Hanson, and V. V. Dobrovitski, Nature 484, 82 (2012).

[26] P. Facchi, D. A. Lidar, and S. Pascazio, Phys. Rev. A 69, 032314 (2004).

[27] K. M. Fonseca-Romero, S. Kohler, and P. Hänggi, Phys. Rev. Lett. 95, 140502 (2005); P. Chen, Phys. Rev. A 73, 022343 (2006).

[28] J. Clausen, G. Bensky, and G. Kurizki, Phys. Rev. Lett. 104, 040401 (2010).

[29] X. Xu, Z. Wang, C. Duan, P. Huang, P. Wang, Y. Wang, N. Xu, X. Kong, F. Shi, X. Rong, and J. Du, Phys. Rev. Lett. 109, 070502 (2012).

[30] F. F. Fanchini, J. E. M. Hornos, and R. d. J. Napolitano, Phys. Rev. A 75, 022329 (2007).

[31] F. F. Fanchini and R. d. J. Napolitano, Phys. Rev. A 76, 062306 (2007).

[32] J. -M. Cai, B. Naydenov, R. Preiffer, L. P. McGuinness, K. D. Jahnke, F. Jelezko, M. B. Plenio, and A. Retzker, New J. Phys. 14, 113023 (2012). 
[33] A. Laraoui and C. A. Meriles, Phys. Rev. B 84, 161403(R) (2011).

[34] C. D. Aiello, M. Hirose, and P. Cappellaro, Nat. Commun. 4, 1419 (2013).

[35] V. V. Mkhitaryan and V. V. Dobrovitski, Phys. Rev. B 89, 224402 (2014).

[36] A. Bermudez, F. Jelezko, M. B. Plenio, and A. Retzker, Phys. Rev. Lett. 107, 150503 (2011).

[37] N. Timoney, I. Baumgart, M. Johanning, A. F. Varón, M. B. Plenio, A. Retzker, and Ch. Wunderlich, Nature 476185 (2011).

[38] A. Bermudez, P. O. Schmidt, M. B. Plenio, and A. Retzker, Phys. Rev. A 85 040302(R) (2012).

[39] M. W. Doherty, N. B. Manson, P. Delaney, F. Jelezko, J. Wrachtrup, and L. C. L. Hollenberg, Phys. Rep. 528, 1 (2013).

[40] A. Albrecht, G. Koplovitz, A. Retzker, F. Jelezko, S. Yochelis, D. Porath, Y. Nevo, O. Shoseyov, Y. Paltiel, and M. B. Plenio, New J. Phys. 16, 093002 (2014).

[41] W. K. Wootters, Phys. Rev. Lett. 80, 2245 (1998).

[42] S. Blanes, F. Casas, J. A. Oteo, J. Ros, Phys. Rep. 470, 151 (2009).

[43] Y. Makhlin, G. Schön, and A. Shnirman, Rev. Mod. Phys. 73, 357 (2001).

[44] D. Bacon, J. Kempe, D. A. Lidar, and K. B. Whaley, Phys. Rev. Lett. 85, 1758 (2000).

[45] J. Kempe, D. Bacon, D. A. Lidar, and K. B. Whaley, Phys. Rev. A 63, 042307 (2001).

[46] D. P. DiVincenzo, D. Bacon, J. Kempe, G. Burkard, and K. B. Whaley, Nature 408, 339 (2000).

[47] J. R. Petta, A. C. Johnson, J. M. Taylor, E. A. Laird, A. Yacoby, M. D. Lukin, C. M. Marcus, M. P. Hanson, and A. C. Gossard, Science 309, 2180 (2005).

[48] M. Anderlini, P. J. Lee, B. L. Brown, J. Sebby-Strabley, W. D. Phillips, and J. V. Porto, Nature 448452 (2007). 